- A survey was carried out in the Summer of 2001 to elicit the views of dentists and professionals complementary to dentistry (PCDs) working within personal dental service (PDS) pilots regarding the role of PCDs (as part of the national evaluation of personal dental services).

- Pilots provided improved flexibility in working patterns for dentists and improved access to training.

- Better working conditions improved recruitment and retention of dentists.

- PCDs had better access to training and development, but remained largely the same as prior to working in the PDS.

- Pilots failed to involve PCDs in the management and development of the pilot sites and failed to use their improved conditions to recruit and retain PCDs.

- There were still concerns regarding the changing role of the CDS for both dentists and PCDs.

\title{
National evaluation of personal dental services: the perspective of dentists and professionals complementary to dentistry
}

\author{
A. C. Hall, ${ }^{1}$ K. B. Hill, ${ }^{2}$ N.Goodwin, ${ }^{3}$ A. J. Morris, ${ }^{4}$ F. J. T. Burke, ${ }^{5}$ Series Editor: K. B. Hill
}

In 2001, research was carried out to investigate the views of dentists and professionals complementary to dentistry on their experiences as part of the personal dental services pilots. This article outlines the main messages from the survey. Before 1997, NHS general dental services could only be delivered by general dental practitioners via a national contract with a nationally negotiated scale of fees. The NHS (Primary Care) Act 1997 allowed for the voluntary establishment of personal dental services (PDS) pilot schemes to test alternative ways of delivering dental services, in particular general dental services, through local contracting arrangements. In the PDS scheme, the contract for dental services was agreed between the commissioners (health authority or primary care trust) and one or more providers of dental services. Since then, the involvement of professionals complementary to dentistry (PCDs) has become an increasingly important issue. This investigation seeks to shed some light on the effectiveness of attempts to integrate the dental team and improve the contribution made by PCDs.

The evaluation of the first wave and second wave PDS pilots sought to examine the extent to which pilots achieved their stated aims and to explore the relationship between what was achieved and why. Particular attention was paid to an exploration of factors facilitating or inhibiting change. Throughout the evaluation it appeared increasingly relevant to assess the views of all the stakeholders working within the PDS pilots. It was recognised that team-working and new skill mix between dental care professionals was needed in the pilots, not only to increase the capacity of NHS dentistry but also to allow dentists to concentrate on more

${ }^{1}$ Lecturer, University of Sheffield, School of Clinical Dentistry, Claremont Crescent Sheffield S10 2TA ${ }^{2 *}$ Lecturer in Behavioural Sciences, ${ }^{4}$ Lecturer in Dental Public Health,

${ }^{5}$ Professor in Primary Dental Care, University of

Birmingham, School of Dentistry, Unit of Dental Public

Health, St Chad's Queensway, Birmingham, B4 6NN,

${ }^{3}$ Lecturer, Health Service Management Centre, Park House,

40 Edgbaston Park Road, Edgbaston, Birmingham, B15 2RT

${ }^{*}$ Correspondence to: Kirsty Hill, University of Birmingham,

School of Dentistry, Primary Dental Care Research Unit, St

Chad's Queensway, Birmingham, B4 6NN

\section{Refereed Paper}

Received 08.05.03; Accepted 28.08.03

doi: 10.1038/sj.bdj.4810783

๑ British Dental Journal 2003; 195: 651-653 complex cases. PDS pilots were the first real opportunity for general dental practitioners (GDPs) to work with a variety of PCDs due to new incentive schemes offered under PDS.

In the main, reports from other key stakeholders were positive about teamwork." However it is important to note that these individuals were involved in the inception and development of their pilot site with a clear view of their destination and the ability to adjust their route should the journey become too uncomfortable.

Other members of the dental team may not have shared those experiences or views, yet they would also be important both as determinants and evaluators of the pilots. Furthermore, the importance of the role of PCDs in relation to the PDS scheme had changed. PCDs had been cited by some pilots as key professionals by whom it was anticipated that their pilot's objectives might be achieved. Therefore, the aim of this part of the evaluation was to assess the development and involvement of dental professionals (184 dentists and 226 PCDs) who were participating in PDS pilot schemes. A subsidiary aim was to compare the development and involvement of dentists and PCDs.

\section{METHOD}

\section{Questionnaire}

The method used to elicit these views was a cross-sectional analytic postal questionnaire. This was sent to all dentists $(n=184)$ and PCDs ( $n=226)$ working within the pilots that formed part of the national evaluation (14 first wave and five second wave). A prepaid reply envelope, covering letter and a copy of the specific pilot objectives were enclosed along with the questionnaire. The questionnaire was designed to gain the views of staff, other than primary stakeholders working with PDS pilots. Questionnaires were sent out in two waves with nonresponders receiving a second opportunity to reply. The questionnaire examined dentists' and PCDs' perceptions of:

- Their role and any perceived change of their role

- Their training and development opportunities

- Their degree of involvement in decisionmaking within the pilot.

The majority of the questions were closed and offered a range of options for

*One pilot now having closed owing to it achieving its objectives. 
the respondents to consider. Open questions allowed the respondents to comment on their PDS pilot in relation to specific topics and finally on the pilot as a whole.

Questions were developed from research themes arising from other aspects of the national evaluation of PDS. Questionnaires were piloted amongst 10 dental and 10 PCDs peers working outside the PDS framework and appropriate amendments made.

Simple descriptive analyses were used to describe and compare the proportions of dentists and PCDs who responded in each category. Where no response was made the participant was coded as 'don't know'. As the evaluation sampled all the staff working in the pilots, hypothesis tests were not necessary. Responses to the open questions were subjected to qualitative descriptive content analysis.

\section{RESPONSE RATE}

Of the questionnaires sent to the dentists, 141 $(81 \%)$ were completed. One hundred and eighty five (85\%) questionnaires were completed and returned by PCDs. Respondents were invited to add comments at the end of each section. Over $43.7 \%$ of dentists made additional comments about their pilot. In contrast, far fewer of the PCDs (7.6\%) took the opportunity to comment. The difference in the frequency of additional comments was viewed by the research team as an interesting finding. It reveals a significant disparity between the two groups when asked to give their wider opinion and perhaps is indicative of the differing roles that remained between dentists and PCDs. Some of the possible reasoning for this is supported by later results in the management section.

\section{EMERGING THEMES FROM THE RESEARCH}

This research gives valuable insight into the use of PCDs within the dental team. In particular it reveals the differing perceptions of dentists and PCDs with regard to their understanding of team involvement. It also shows that whilst PCDs may be used in greater numbers today, their role and position in the dental team largely remains the same as previously.

The results of the study fall into five main themes:

- Roles and responsibility

- Role of the CDS

- Recruitment

- Management

- Pilot progress

\section{Role and responsibility}

Half of all dentists (50.0\%) reported that their roles and responsibilities had changed since becoming involved in the pilots but this change, on the whole had been a positive one. Also, dentists reported that job satisfaction was found not to relate to volume of work but was closely linked to role diversity and effective team cohesion.

The comments below formed a common theme in the responses:

'Working in a PDS pilot is an enjoyable way to work in the dental field. There is a very strong sense of working as part of a team, which is committed to both the job and NHS dentistry'

And: 'I am enjoying working in the PDS pilot; I am able to provide relevant services to patients. This may often mean I spend more time giving advice and education to patients, which would not have been [possible] under GDS'.

Dentists also reported negative aspects of PDS which included the increase in the amount of paperwork, dealing with the Dental Practice Board (DPB), patients' charges and the increase in patient load. There were strong views held particularly by former CDS dentists, that their specialised role was being diminished.

In contrast, $34.6 \%$ of PCDs felt that their role had changed. However, PCDs reporting change had generally worked formerly in the CDS and much of the change reported centred on collection of patient charges rather than a change in role or duties. The remainder of PCDs who reported change commented on increased administration levels, in line with their dentist colleagues. Reports from dentists that their role had become more diverse and afforded them greater job satisfaction were not mirrored by PCDs. PCDs also did not report greater flexibility in working patterns.

Examples were noted in which individual pilots had sought to re-evaluate the way in which staff were used and to incorporate them more wholeheartedly into the management structure. Some pilot sites had also introduced the use of therapists, or in one case anaesthetists, to support the recruitment crisis in dentistry and meet the specific dental needs in their community. However, where a specific need was not being met and outside the new use of therapists in a GDS style setting, the change in PCDs roles was not greatly in evidence. Despite the PDS pilot scheme providing an opportunity to develop the use of skill mix within the pilot context, it appeared that the use of skill mix remained in its infancy. Traditional cultural boundaries remained, and, despite the scheme's ability to embrace differing methods of working patterns for dentists and a change in remuneration, the role of PCDs remains static.

\section{Role of CDS}

In the early stages of the evaluation it was reported ${ }^{1}$ that tensions developed in some pilots between different professionals who were not accustomed to working together, and between different services which had traditionally operated within boundaries. At the outset, these contrasting qualities had resulted in a lack of cohesion in some pilot teams and in turn acted as inhibitors to the pilot's overall development.

Dentists reported that the culture change from the CDS to PDS had been particularly difficult, especially early on. One practitioner commented: '...there were ripples of discontentment in the early stages but this has disappeared now'.

The culture change for CDS staff was immense. One practitioner reported: 'I feel the PDS scheme is generally damaging the care of special needs patients. We are just becoming an NHS emergency service'. This comment was reiterated throughout the former CDS, that emergency patients were being given priority over special needs patients. Other comments suggest that the skills that CDS dentists have developed would diminish if their role was not protected. However, one practitioner suggested that seeing a different mix of patients did in fact increase the dentists' basic skills rather than reducing them.

Dentists had to switch from being completely focused on treatment of patients with special needs to a more mainstream service involving Dental Practice Board (DPB) paperwork, changing the way information was recorded and collecting patient charges. Many of the former CDS found these changes an enormous hurdle to overcome and some staff left the service as a result.

Former CDS PCDs also highlighted misgivings. They too had found the cultural change difficult and anxieties for them centred on the collection of patient charges. Many felt uncomfortable with this role.

Despite a large cultural shift, PCDs did not report any hostility between their role and that of the dentists, a theme that had been anticipated with the increasing use of therapists and hygienists in particular. However this finding emerged as less surprising as it became apparent how little the role of PCDs had changed as a result of PDS and therefore the need for any potential team adjustment was removed.

\section{Recruitment}

The progress of the PDS scheme had been pressured by the short supply and lack of availability of dentists. Dentists reported examples of poaching staff where new pilot sites were able to offer enhanced packages. More than one quarter of dentists had been offered a financial incentive to join the pilot (27.5\%). Whilst this 'poaching' of staff was clearly an undermining factor in team cohesion and pilot development, it highlights the attractiveness of PDS pilots. Additionally, the current demand for dentists in the private sector and the high salaries that these 
posts offer, had also lured those dentists to work elsewhere.

Given that one of the potential successes of the PDS system was its potential to improve access and provide more appropriate dental services to areas with acute oral health needs, overcoming recruitment and retention difficulties remained a key inhibitor and potential destabilising factor for such pilots. Nevertheless, it was hoped by many in such areas that the professional opportunities available to dentists and other staff working in such PDS pilots might outweigh any downsides for certain employees. Pilots had been imaginative in making posts attractive to dentists; in particular dedicated staff training programmes and the different style and culture of the PDS dental delivery system were regarded by the dentists as key strengths that helped to attract and retain dental professionals. However, many PDS pilots reported continual difficulties with recruitment and retention of dentists and other staff.

An increasing use of skill mix in the PDS pilots had been a focus of several bids for the PDS scheme to help alleviate the pressure on the recruitment crisis in dentistry. It was anticipated that staff levels could be increased through more imaginative use of PCDs including the use of therapists and improving the roles of nursing staff. Better team dynamics could then in turn lead to increased work capacity therefore enabling the registration of a greater number of patients.

However, despite pressure on the availability of staff for both dentists and PCDs, the PDS pilot sites had not appeared to take an active approach to attracting PCD staff in the same way as they had attempted to attract dentists. Far fewer incentives had been offered to PCDs to encourage them to join the pilot scheme. Only $4.3 \%$ of PCDs had been offered a financial incentive to join the PDS scheme as compared with 27.5\% of dentists. Almost twice as many dentists than PCDs had been offered nonfinancial incentives, such as training and development (22.5\% vs. $12.4 \%)$. This was surprising as increasing the use of skill mix was viewed at the initial stages of some pilots as forming an integral part of meeting their objectives. The focus still remained very much on recruiting dentists and not on recruiting PCDs. Interestingly PCDs had received significant levels of training. Similar numbers of dentists and PCDs (12.7\% and $13.0 \%$ respectively) had attended courses that had led to a professional qualification. However, even where PCDs had received high levels of training, this access to training was not perceived by the PCDs as being an incentive, possibly because access to training had not been used by the pilots as an effective recruitment tool. It could be

Table 1 PCD and dentist team involvement

\begin{tabular}{|c|c|c|c|c|c|}
\hline & Always (\%) & Sometimes (\%) & Rarely (\%) & Never (\%) & Don't Know (\%) \\
\hline \multicolumn{6}{|c|}{ How often do you attend review meetings? } \\
\hline Dentists & 50.0 & 14.1 & 11.3 & 22.5 & 2.1 \\
\hline PCDs & 18.3 & 18.3 & 8.3 & 50.8 & 4.3 \\
\hline \multicolumn{6}{|c|}{ How often do you attend PDS away-days and events? } \\
\hline Dentists & 29.6 & 13.4 & 6.3 & 45.8 & 4.9 \\
\hline PCDs & 11.9 & 7.6 & 7.6 & 65.9 & 7.0 \\
\hline \multicolumn{6}{|c|}{ How often do you comment on discussion documents? } \\
\hline Dentists & 31.7 & 34.5 & 14.8 & 17.6 & 1.4 \\
\hline PCDs & 5.9 & 15.7 & 16.8 & 55.1 & 6.5 \\
\hline
\end{tabular}

Table 2 PCD and dentists management involvement $\begin{array}{lllll}\text { Very good }(\%) & \text { Good }(\%) & \text { Satisfactory }(\%) & \text { Poor }(\%) & \text { Very poor }(\%)\end{array}$

How would you assess the feedback mechanism that exists between the clinical lead of the pilot and you?

\begin{tabular}{lcccccc} 
Dentists & 34.5 & 26.1 & 22.5 & 10.6 & 2.8 & 3.5 \\
PCDs & 11.4 & 23.5 & 30.3 & 17.4 & 12.5 & 4.9 \\
\hline & & & & & & No \\
& Great (\%) & Significant (\%) & Moderate (\%) & Little (\%) & No (\%) & Don't know (\%) \\
\hline
\end{tabular}

How would you describe your involvement in the PDS pilot's decision-making process?

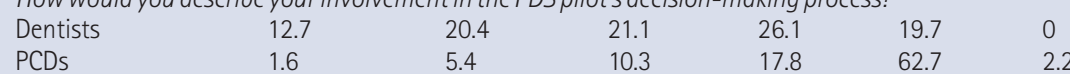

concluded that pilots did have better training opportunities for PCDs but had not viewed their recruitment as a sufficient priority to make the most of the conditions they had to offer. This is perhaps indicative of how the PCDs' role was viewed within the pilot context and belies a more reticent attitude to PCD recruitment and retention than had previously been expected.

\section{Management}

As can be seen from Tables 1 and 2, most dentists had been active contributors to the management and development of the pilot and that had added to the satisfaction they had found in their roles. In contrast, however, PCDs had rarely contributed to management decisions or even been regular attendees at team meetings. Although this was not negatively reported by many PCDs who seemed to accept their lack of involvement, it may be considered disappointing to find that PCD staff had not been asked or encouraged to contribute, in some cases, their years of experience to the development and management of their pilot. It appeared that for the most part, PCDs only contributed their skills to the dental team but not their opinions.

\section{Pilot progress}

The proportion of dentists who thought their pilot was progressing very well, well, average, poor and don't know was 31.0\%, $35.2 \%, 21.8 \%, \quad 4.9 \%, \quad 7.1 \%$ respectively, whereas the proportions for PCDs were $32.4 \%, \quad 24.8 \%, \quad 28.1 \%, \quad 7.5 \%$ and $7.2 \%$ respectively. Most dentists reported that becoming involved in a PDS pilot scheme had improved their overall job satisfaction.

Only the two practitioners who reported that they had not seen the objectives of the pilots prior to receiving the questionnaire suggested that PDS was not successful and that the money could be used more efficiently elsewhere. Possibly, access to pilot information could have led to a more positive attitude, however this cannot be confidently concluded.

Interestingly, despite a low level of involvement in management and development of the pilot, most PCDs reported that they were positive about the progression of their pilots and felt that good progress had been made toward achieving the pilot's objectives. During the course of the completion of the questionnaires, far fewer PCDs availed themselves of the opportunity to give their opinion on the scheme, a stark contrast to their dentist colleagues.

\section{SUMMARY}

For most dentists, working conditions in their pilot were very good. There was a significant improvement in working hours, access to training and management involvement. Concerns over the demise of the former CDS persisted and this area needs further investigation as the pilots develop.

Many pilots had failed to consider how PCDs could help to overcome difficulties faced within the pilots, or how a change in their role could facilitate recruitment. PCDs were still contributing their clinical skills but rarely their general experience to pilot management and development. This seemed to meet the expectation of most PCDs who were satisfied with their level of contribution and the feedback they received, but falls somewhat short of the opportunities within PDS to create a more dynamic multi-disciplinary scheme.

This work was undertaken by the University of Birmingham who received funding from the Department of Health; the views expressed in this publication are those of the authors and not necessarily those of the Department of Health.

1. Smith J, McLeod H, Goodwin N, Morris J, Hill K, Hall A, Bradnock G, Ham C. New models of NHS dental care: interim report of the national evaluation of Personal DentalServices. University of Birmingham: Health Services Management Centre, December 2000. 